\title{
A DIFFUSIVE SVEIR EPIDEMIC MODEL WITH TIME DELAY AND GENERAL INCIDENCE*
}

\author{
Jinling ZHOU (周金玲) Xinsheng MA (马新生) \\ Department of Mathematics, Zhejiang International Studies University, Hangzhou 310023, China \\ E-mail: jlzhou@amss.ac.cn; xsma@zisu.edu.cn \\ $Y u$ YANG $(\text { 杨瑜 })^{\dagger}$ \\ School of Statistics and Mathematics, Shanghai Lixin University of Accounting and Finance, \\ Shanghai 201209, China \\ E-mail:yangyu@lixin.edu.cn \\ Tonghua ZHANG (张同华) \\ Department of Mathematics, Swinburne University of Technology, Hawthorn, Victoria 3122, Australia \\ E-mail: tonghuazhang@swin.edu.au
}

\begin{abstract}
In this paper, we consider a delayed diffusive SVEIR model with general incidence. We first establish the threshold dynamics of this model. Using a Nonstandard Finite Difference (NSFD) scheme, we then give the discretization of the continuous model. Applying Lyapunov functions, global stability of the equilibria are established. Numerical simulations are presented to validate the obtained results. The prolonged time delay can lead to the elimination of the infectiousness.
\end{abstract}

Key words SVEIR model; vaccination; Lyapunov function; nonstandard finite difference method

2010 MR Subject Classification 34F05; 60H10; 93E15; 92B05

\section{Introduction}

Vaccination is an effective way of controlling the transmission of infectious diseases such as tuberculosis and tetanus etc.. Thus, many countries provide routine vaccination against all of these diseases. However, vaccine-induced immunity may wane as time goes on. To better understand this phenomenon, mathematical models have been developed. Kribs-Zaleta and Velasco-Hernández [1] considered an SIS disease model with vaccination. Arino et al. [2] investigated an SIRS model with vaccination. Li et al. [3] indicated that vaccine effectiveness plays a key role in disease prevention and control. To describe vaccination strategy, Liu et al. [4] considered SVIR epidemic models. Let $S, V, I$ and $R$ be the susceptible, vaccinated,

${ }^{*}$ Received March 23, 2020; revised October 1, 2020.

${ }^{\dagger}$ Corresponding author 
infectious and recovered individuals, respectively. Furthermore, Li and Yang [5] proposed the following model for $t>0$ :

$$
\left\{\begin{array}{l}
\frac{\mathrm{d} S}{\mathrm{~d} t}=\mu p A-\mu S-\beta S I-\alpha S+\eta V, \\
\frac{\mathrm{d} V}{\mathrm{~d} t}=\mu q A+\alpha S-\sigma \beta V I-(\mu+\eta) V, \\
\frac{\mathrm{d} I}{\mathrm{~d} t}=\beta I(S+\sigma V)-(\gamma+\mu+\delta) I, \quad \frac{\mathrm{d} R}{\mathrm{~d} t}=\gamma I-\mu R .
\end{array}\right.
$$

Here $\mu$ and $A$ represent the death rate and the birth rate, respectively. $q<1$ denotes the fraction of the vaccinated newborns, $p$ is the unvaccinated newborns, $0<\sigma<1$ represents that the vaccine is not completely effective, $\beta$ is the transmission coefficient of the susceptible, $\gamma$ is the recovery rate, $\delta$ is the per capita disease-induced death rate. The susceptible population is vaccinated at a constant rate $\alpha$ and the vaccine-induced immunity wanes at rate $\eta$. Li and Yang discussed the global dynamics of system (1.1) by applying Lyapunov functions.

As seen from the existing models, incidence rates play a very important role in determining model dynamics; for example, the bilinear incidence rate is applicable to Hand-Foot-and-Mouth disease [6], H5N1 [7] and SARS [8], but not to sexually transmitted diseases [9]. To model the effect of behavioural changes, Liu et al. [10] proposed an incidence rate $\frac{\beta S I^{p}}{1+\alpha I^{q}}$. To model the cholera epidemics in Bari, Capasso and Serio [11] considered the incidence rate $p=q=1$. Due to a diseases latency, or factors of immunity, infection processes are not instantaneous. Hence, time delay is important in studying infectious disease dynamics. Hattaf et al. [12] studied a delayed SIR model with general incidence. Wang et al. [13] proposed a delayed SVEIR model with nonlinear incidence. Recently, Hattaf [14] proposed a generalized viral infection model with multi-delays and humoral immunity. For more works on delayed epidemic models with vaccination, we refer readers to [15-19].

All of the above mentioned works are location independent, but location-dependent phenomenon are not uncommon in mathematical biology (see [20-22]). Webby [23] pointed out that infectious cases can first be found at one location and can then spread to other areas. Therefore, it is interesting to study epidemic models with spatial diffusion. Xu and Ai [24] considered an influenza disease model with spatial diffusion and vaccination. Abdelmalek and Bendoukha [25] proposed a diffusive SVIR epidemic model allowing continuous immigration of all classes of individuals. Xu et al. [26] discussed a vaccination model with spatial diffusion and nonlinear incidence.

Let $\Omega$ be a bounded domain in $\mathbb{R}^{n}$ with smooth boundary $\partial \Omega$. Let $D_{i}(i=1,2,3,4,5)$ be the diffusion rate and $\Delta$ be the Laplace operator. Then, motivated by the aforementioned works, particularly $[13,23]$, we study the delayed SVEIR model with spatial diffusion as follows:

$$
\left\{\begin{array}{c}
\frac{\partial S(x, t)}{\partial t}=D_{1} \Delta S+\mu p A-S f(I)-(\mu+\alpha) S, x \in \Omega, t>0, \\
\frac{\partial V(x, t)}{\partial t}=D_{2} \Delta V+\mu q A+\alpha S-g(I) V-(\beta+\mu) V, x \in \Omega, t>0, \\
\frac{\partial E(x, t)}{\partial t}=D_{3} \Delta E+f(I) S+g(I) V-e^{-\mu \tau}[f(I(t-\tau)) S(t-\tau) \\
\quad+g(I(t-\tau)) V(t-\tau)]-\mu E, x \in \Omega, t>0, \\
\frac{\partial I(x, t)}{\partial t}=D_{4} \Delta I+e^{-\mu \tau}[f(I(t-\tau)) S(t-\tau)+g(I(t-\tau)) V(t-\tau)] \\
\quad-(\delta+\gamma+\mu) I, x \in \Omega, t>0, \\
\frac{\partial R(x, t)}{\partial t}=D_{5} \Delta R+\beta V+\delta I-\mu R, x \in \Omega, t>0, \\
\frac{\partial S}{\partial \vec{n}}=\frac{\partial V}{\partial \vec{n}}=\frac{\partial E}{\partial \vec{n}}=\frac{\partial I}{\partial \vec{n}}=\frac{\partial R}{\partial \vec{n}}=0, \quad x \in \partial \Omega, t>0 .
\end{array}\right.
$$


Here, $\tau$ represents the latent period of the disease. The other parameters are as described for system (1.1). Denote by $\vec{n}$ the outward unit normal vector of $\partial \Omega$ as in $[20,21]$. We further consider model (1.2) with initial condition

$$
\begin{aligned}
& S(x, \theta)=\varphi_{1}(x, \theta), V(x, \theta)=\varphi_{2}(x, \theta), E\left((x, \theta)=\varphi_{3}(x, \theta),\right. \\
& I(x, \theta)=\varphi_{4}(x, \theta), R(x, \theta)=\varphi_{5}(x, \theta), \quad x \in \bar{\Omega}, \theta \in[-\tau, 0],
\end{aligned}
$$

where $\varphi_{i}(i=1,2,3,4,5)$ are uniformly continuous and bounded. Functions $g$ and $f$ satisfy $g(0)=f(0)=0$ and

(H1) for $I>0, g(I)>0$ and $f(I)>0$;

(H2) for $I \geq 0, g^{\prime}(I)>0$ and $f^{\prime}(I)>0, g^{\prime \prime}(I) \leq 0$ and $f^{\prime \prime}(I) \leq 0$.

Epidemiologically, (H1) means that individuals are positive. (H2) implies that the incidences of $S f(I)$ and $V g(I)$ become faster with an increase in the number of the infectious individuals. However, the per capita infection rate will slow down because of a certain inhibition effect, since $g^{\prime \prime}(I), f^{\prime \prime}(I) \leq 0$ imply that $\left(\frac{f(I)}{I}\right)^{\prime},\left(\frac{g(I)}{I}\right)^{\prime}<0$. For example, the commonly used nonlinear incidence function $\frac{x}{1+\alpha x}(\alpha>0)$ satisfies both (H1) and (H2).

In this study, in addition to model (1.2), we will also investigate the discrete analogue, due to the fact that epidemiological data is usually collected daily, monthly, or even yearly, but not continuously. Hence, it is more reasonable to use a discrete model to study the transmission mechanism of infectious disease. Furthermore, it is an interesting problem as to whether or not a selected difference scheme can preserve the positivity, boundedness and global stability for the corresponding continuous model. In this regard, some researchers have applied the NSFD scheme proposed by Mickens [27] to discuss the dynamical behaviors of different epidemic models ([28-37]).

The rest of the paper is organized as follows: in Section 2, we establish the global dynamics of the continuous model (1.2). In Section 3, we derive the discretization of (1.2) by the NSFD scheme and establish the positivity and boundedness of the solution. By using discrete Lyapunov functionals, we discuss the global stability of the equilibria of the discretised model in Section 4. This is then followed by numerical simulations in Section 5 to illustrate the obtained results.

\section{The Continuous Model}

From system (1.2), we only discuss the following system:

$$
\left\{\begin{aligned}
& \frac{\partial S(x, t)}{\partial t}=D_{1} \Delta S+\mu p A-S f(I)-(\mu+\alpha) S, x \in \Omega, t>0 \\
& \frac{\partial V(x, t)}{\partial t}= D_{2} \Delta V+\mu q A+\alpha S-g(I) V-(\beta+\mu) V, x \in \Omega, t>0 \\
& \frac{\partial I(x, t)}{\partial t}= D_{4} \Delta I+e^{-\mu \tau}[f(I(t-\tau)) S(t-\tau)+g(I(t-\tau)) V(t-\tau)] \\
&-(\delta+\gamma+\mu) I, x \in \Omega, t>0 \\
& \frac{\partial S}{\partial \vec{n}}=\frac{\partial V}{\partial \vec{n}}=\frac{\partial I}{\partial \vec{n}}=0, \quad x \in \partial \Omega, t>0
\end{aligned}\right.
$$

with

$$
S(x, \theta)=\varphi_{1}(x, \theta), V(x, \theta)=\varphi_{2}(x, \theta), I(x, \theta)=\varphi_{4}(x, \theta), \quad x \in \bar{\Omega}, \theta \in[-\tau, 0] .
$$




\subsection{Threshold dynamics}

In this section we assume that $D_{1}=D_{2}=D_{4}=D$. Let $\mathbb{X}:=C\left(\bar{\Omega}, \mathbb{R}^{3}\right)$ be a Banach space with the supremum form $\|\cdot\|_{\mathbb{X}}$ and $\tau>0$. Let $\mathbf{C}_{\tau}:=C([-\tau, 0], \mathbb{X})$ with the form $\|\tilde{\phi}\|:=$ $\max _{\theta \in[-\tau, 0]}\|\tilde{\phi}(\theta)\|_{\mathbb{X}}, \forall \tilde{\phi} \in \mathbf{C}_{\tau}$. Define $\mathbb{X}^{+}:=C\left(\bar{\Omega}, \mathbb{R}_{+}^{3}\right)$ and $\mathbf{C}_{\tau}^{+}:=C\left([-\tau, 0], \mathbb{X}^{+}\right)$. Then $\left(\mathbb{X}, \mathbb{X}^{+}\right)$ and $\left(\mathbf{C}_{\tau}, \mathbf{C}_{\tau}^{+}\right)$are strongly ordered spaces. For any given function $\tilde{\phi}(t):[-\tau, \varsigma) \rightarrow \mathbb{X}(\varsigma>0)$, we denote $\tilde{\phi}_{t} \in \mathbf{C}_{\tau}$ by

$$
\tilde{\phi}_{t}(\theta)=\tilde{\phi}(t+\theta), \quad \forall \theta \in[-\tau, 0] .
$$

Let $\mu_{1}=\alpha+\mu, \mu_{2}=\beta+\mu$ and $\mu_{3}=\delta+\gamma+\mu$. Define $\tilde{T}_{j}(t): C(\bar{\Omega}, \mathbb{R}) \rightarrow C(\bar{\Omega}, \mathbb{R})$ by the $C_{0}$ semigroups with $D \Delta-\mu_{j}(j=1,2,3)$. With $\forall t \geq 0$ and $\tilde{\phi} \in C(\bar{\Omega}, \mathbb{R})$, we have

$$
\tilde{T}_{j}(t) \tilde{\phi}(x)=e^{-\mu_{j} t} \int_{\Omega} \tilde{\Gamma}(x, t, s) \tilde{\phi}(s) \mathrm{d} s, j=1,2,3,
$$

where $\tilde{\Gamma}$ is the Green function associated with $D \Delta$. According to [35, Corollary 4], $\tilde{T}_{j}(t)$ are strongly positive and compact $(j=1,2,3, \forall t>0)$. Taking $\forall x \in \bar{\Omega}$ and $\tilde{\phi}=\left(\phi_{1}, \phi_{2}, \phi_{3}\right) \in \mathbb{X}^{+}$, let $\tilde{F}=\left(F_{1}, F_{2}, F_{3}\right): \mathbb{X}^{+} \rightarrow \mathbb{X}$ be

$$
\begin{aligned}
& F_{1}(\tilde{\phi})(x)=\mu p A-f\left(\phi_{3}(x, 0)\right) \phi_{1}(x, 0)-(\alpha+\mu) \phi_{1}(x, 0), \\
& F_{2}(\tilde{\phi})(x)=\mu q A+\alpha \phi_{1}(x, 0)-g\left(\phi_{3}(x, 0)\right) \phi_{2}(x, 0)-(\beta+\mu) \phi_{2}(x, 0), \\
& F_{3}(\tilde{\phi})(x)=e^{-\mu \tau}\left(f\left(\phi_{3}(x,-\tau)\right) \phi_{1}(x,-\tau)+g\left(\phi_{3}(x,-\tau)\right) \phi_{2}(x,-\tau)\right)-(\delta+\gamma+\mu) \phi_{3}(x, 0) .
\end{aligned}
$$

Then, system (2.1) can be rewritten as

$$
\tilde{Z}(x, t)=\tilde{T}(t) \tilde{\phi}(x)+\int_{0}^{t} \tilde{T}(t-s) \tilde{F}(\tilde{Z}(x, s)) \mathrm{d} s,
$$

where $\tilde{Z}(x, t)=((S(x, t), V(x, t), I(x, t))$ and

$$
\tilde{T}=\left(\begin{array}{ccc}
\tilde{T}_{1} & 0 & 0 \\
0 & \tilde{T}_{2} & 0 \\
0 & 0 & \tilde{T}_{3}
\end{array}\right) .
$$

Theorem 2.1 System $(2.1)-(2.2)$ admits a unique solution $\tilde{T}(\cdot, t, \tilde{\phi})$ on $[0, \infty)$ satisfying $\tilde{Z}(\cdot, 0, \tilde{\phi})=\tilde{\phi}$ for all $\tilde{\phi} \in \mathbb{X}^{+}$. Given $\forall x \in \bar{\Omega}$ and $t \geq 0$, the semiflow

$$
\Theta(t) \tilde{\phi}=(S(., t, \tilde{\phi}), V(., t, \tilde{\phi}), I(., t, \tilde{\phi}))
$$

is point dissipative.

Proof Taking $\forall \tilde{\phi} \in \mathbb{X}^{+}$and $k>0$ (sufficiently small), we have

$$
\begin{aligned}
\tilde{\phi}(x, 0)+k F(\tilde{\phi})(x)= & \left(\begin{array}{c}
\phi_{1}(x, 0)+k\left(\mu p A-\phi_{1}(x, 0) f\left(\phi_{3}(x, 0)\right)-(\mu+\alpha) \phi_{1}(x, 0)\right) \\
\phi_{2}(x, 0)+k\left(\mu q A+\alpha \phi_{1}(x, 0)-\phi_{2}(x, 0) g\left(\phi_{3}(x, 0)\right)-(\mu+\beta) \phi_{2}(x, 0)\right) \\
\phi_{3}(x, 0)+k\left(e^{-\mu \tau}\left(f\left(\phi_{3}(x,-\tau)\right) \phi_{1}(x,-\tau)+g\left(\phi_{3}(x,-\tau)\right)\right) \phi_{2}(x,-\tau)\right. \\
\left.-(\mu+\delta+\gamma) \phi_{3}(x, 0)\right)
\end{array}\right) \\
\geq & \left(\begin{array}{c}
\phi_{1}(x, 0)\left(1-k\left((\alpha+\mu)+f\left(\phi_{3}(x, 0)\right)\right)\right. \\
\phi_{2}(x, 0)\left(1-k\left((\mu+\beta)+g\left(\phi_{3}(x, 0)\right)\right)\right. \\
\phi_{3}(x, 0)(1-k(\mu+\delta+\gamma))
\end{array}\right) \geq\left(\begin{array}{l}
0 \\
0 \\
0
\end{array}\right) .
\end{aligned}
$$


The above inequality implies that

$$
\lim _{k \rightarrow 0^{+}} \operatorname{dist}\left(\tilde{\phi}+k F(\tilde{\phi}), \mathbf{C}_{\tau}^{+}\right)=0 .
$$

According to [38, Corollary 4], one can derive that system (2.1) has a unique mild solution $\tilde{Z}(\cdot, t, \tilde{\phi}) \in \mathbb{X}^{+}$for $t \in\left[0, \tau_{\tilde{\phi}}\right)$.

Define $W(x, t)=S(x, t)+V(x, t)$. Then, we can get

$$
\frac{\partial W(x, t)}{\partial t}=D \triangle W+\mu A-d_{1} W
$$

where $d_{1}=\min \{\mu+\alpha, \mu+\beta\}$. Then, $S(x, t)$ and $V(x, t)$ are bounded on $\left[0, \tau_{\tilde{\phi}}\right)$ by using a comparison principle. Define $\tilde{G}(x, t)=S(x, t-\tau)+V(x, t-\tau)+e^{\mu \tau} I(x, t)$. Then, we have

$$
\frac{\partial \tilde{G}(x, t)}{\partial t} \leq D \triangle \tilde{G}+\mu A-d_{2} \tilde{G}(x, t)
$$

where $d_{2}=\min \{\mu, \mu+\beta, \mu+\delta+\gamma\}$. Thus, $\tilde{G}(x, t)$ are bounded on $\left[0, \tau_{\tilde{\phi}}\right)$, by the comparison principle. This implies that $I(x, t)$ are also bounded on $\left[0, \tau_{\tilde{\phi}}\right)$. The remaining proofs are similar to Theorem 2.1 of Zhou et al. [34], which we omit here.

\section{$2.2 \quad$ Existence of equilibria}

Define

$$
S_{0}=\frac{\mu p A}{\mu+\alpha}, \quad V_{0}=\frac{\mu A(q(\mu+\alpha)+\alpha p)}{(\mu+\alpha)(\mu+\beta)} .
$$

Then, $E_{0}\left(S_{0}, V_{0}, 0\right)$ is the disease-free equilibrium of system (2.1). The basic reproduction number is

$$
\mathcal{R}_{0}=\frac{S_{0} f^{\prime}(0)}{e^{\mu \tau}(\mu+\delta+\gamma)}+\frac{V_{0} g^{\prime}(0)}{e^{\mu \tau}(\mu+\delta+\gamma)} .
$$

The endemic equilibrium should satisfy

$$
\left\{\begin{array}{l}
\mu p A-f(I) S-(\alpha+\mu) S=0, \\
\mu q A+\alpha S-g(I) V-(\beta+\mu) V=0, \\
g(I) V+f(I) S-e^{\mu \tau} I(\gamma+\mu+\delta)=0 .
\end{array}\right.
$$

Theorem 2.2 If $\mathcal{R}_{0}<1$, then system (2.1) has a unique disease-free equilibrium $E_{0}\left(S_{0}\right.$, $\left.V_{0}, 0\right)$; if $\mathcal{R}_{0}>1$, then system $(2.1)$ has a unique endemic equilibrium $E^{*}\left(S^{*}, V^{*}, I^{*}\right)$ with $S^{*}=\frac{\mu p A}{\mu+\alpha+f\left(I^{*}\right)}$ and $V^{*}=\frac{\mu q A\left(\mu+\alpha+f\left(I^{*}\right)\right)+\alpha \mu p A}{\left(\mu+\beta+g\left(I^{*}\right)\right)\left(\mu+\alpha+f\left(I^{*}\right)\right)}$, except for $E_{0}$.

Proof When $\mathcal{R}_{0}<1$, the result is obvious.

According to the first two equations of (2.4), one can get

$$
S=\frac{A \mu p}{f(I)+\mu+\alpha}, \quad V=\frac{\mu q A(\alpha+f(I)+\mu)+\alpha \mu p A}{(\beta+g(I)+\mu)(f(I)+\mu+\alpha)} .
$$

Then, we have

$$
h(I)=\frac{\mu p A}{f(I)+\mu+\alpha} f(I)+\frac{\mu q A(\alpha+f(I)+\mu)+\alpha \mu p A}{(g(I)+\mu+\beta)(f(I)+\mu+\alpha)} g(I)-e^{\mu \tau}(\delta+\gamma+\mu) I .
$$

Obviously, $h(+\infty)=-\infty$ and $h(0)=0$. It follows from $h^{\prime}(0)>0$ that $h(I)=0$ has at least one positive solution denoted by $I^{*}$, where

$$
h^{\prime}(0)=-e^{\mu \tau}(\delta+\gamma+\mu)+\frac{\mu p A f^{\prime}(0)}{\alpha+\mu}+\frac{\mu q A(\alpha+\mu)+\alpha \mu p A}{(\alpha+\mu)(\beta+\mu)} g^{\prime}(0)
$$




$$
=e^{\mu \tau}(\gamma+\mu+\delta)\left(\mathcal{R}_{0}-1\right)>0 .
$$

This is equivalent to $\mathcal{R}_{0}>1$. Thus, (2.4) has at least one positive solution with

$$
S^{*}=\frac{\mu p A}{\mu+\alpha+f\left(I^{*}\right)}, \quad V^{*}=\frac{\mu q A\left(\mu+\alpha+f\left(I^{*}\right)\right)+\alpha \mu p A}{\left(\mu+\beta+g\left(I^{*}\right)\right)\left(\mu+\alpha+f\left(I^{*}\right)\right)} .
$$

We now prove that the endemic equilibrium is unique. Note that

$$
\begin{aligned}
h^{\prime}(I)= & \frac{\mu p A(\alpha+\mu)}{(f(I)+\mu+\alpha)^{2}} f^{\prime}(I)-\frac{\alpha \mu p A}{(f(I)+\mu+\alpha)^{2}(\beta+g(I)+\mu)} g(I) f^{\prime}(I) \\
& +\left(\mu q A+\frac{A \alpha \mu p}{\alpha+f(I)+\mu}\right) \frac{(\mu+\beta)}{(\beta+g(I)+\mu)^{2}} g^{\prime}(I)-e^{\mu \tau}(\delta+\gamma+\mu),
\end{aligned}
$$

and

$$
\begin{aligned}
h^{\prime \prime}(I)= & \left(\mu p A(\mu+\alpha)-\frac{A p \alpha \mu g(I)}{g(I)+\mu+\beta}\right) \frac{(\alpha+\mu+f(I)) f^{\prime \prime}(I)-2\left(f^{\prime}(I)\right)^{2}}{(\alpha+\mu+f(I))^{3}} \\
& +(\mu+\beta)\left(\mu q A+\frac{\alpha \mu p A}{\mu+\alpha+f(I)}\right) \frac{g^{\prime \prime}(I)(\beta+\mu+g(I))-2\left(g^{\prime}(I)\right)^{2}}{(\beta+\mu+g(I))^{3}} \\
& -\frac{2 \alpha \mu p A(\beta+\mu) g^{\prime}(I) f^{\prime}(I)}{(\alpha+\mu+f(I))^{2}(\beta+\mu+g(I))^{2}} \\
= & \mu p A\left(\frac{(\beta+\mu) \alpha}{\beta+\mu+g(I)}+\mu\right) \frac{(\alpha+\mu+f(I)) f^{\prime \prime}(I)-2\left(f^{\prime}(I)\right)^{2}}{(\alpha+\mu+f(I))^{3}} \\
& +(\mu+\beta)\left(\mu q A+\frac{\alpha \mu p A}{\mu+\alpha+f(I)}\right) \frac{g^{\prime \prime}(I)(\beta+\mu+g(I))-2\left(g^{\prime}(I)\right)^{2}}{(\beta+\mu+g(I))^{3}} \\
& -\frac{2 \alpha \mu p A(\beta+\mu)}{(\alpha+\mu+f(I))^{2}(\beta+\mu+g(I))^{2}} g^{\prime}(I) f^{\prime}(I) .
\end{aligned}
$$

By (H2), we know that $h^{\prime \prime}(I)<0$ for $I>0$. If there exists more than one positive equilibrium, then there must exist a point $E_{*}\left(S_{*}, V_{*}, I_{*}\right)$ such that $h^{\prime \prime}\left(I_{*}\right)=0$. We obtain a contradiction.

\subsection{Local stability}

Let $0=\mu_{0}<\mu_{i}<\mu_{i+1}$ be the eigenvalues of $-\Delta$ on $\Omega$, and $E\left(\mu_{i}\right)$ be the space of eigenfunctions with $\mu_{i}(i=1,2 \ldots)$. Then, we define the orthonormal basis of $E\left(\mu_{i}\right)(i=1,2 \ldots)$ by $\left\{\phi_{i j}: j=1,2, \ldots, \operatorname{dim} E\left(\mu_{i}\right)\right\}$ as follows:

$$
\mathbb{X}=\bigoplus_{i=1}^{\infty} \mathbb{X}_{i}, \quad \mathbb{X}_{i}=\bigoplus_{i=1}^{\operatorname{dim} E\left(\mu_{i}\right)} \mathbb{X}_{i j}
$$

Here, $\mathbb{X}_{i j}=\left\{\mathbf{c} \phi_{i j}: \mathbf{c} \in \mathbb{R}^{3}\right\}$. In a fashion similar to [20, Theorem 3.1], one gets the following result:

Theorem 2.3 If $\mathcal{R}_{0}<1$, then $E_{0}$ of system (2.1) is locally asymptotically stable.

Proof Linearizing system (2.1) at $E_{0}$, we get

$$
\frac{\partial \tilde{Z}(x, t)}{\partial t}=\mathcal{S} \triangle \tilde{Z}(x, t)+\mathcal{A} \tilde{Z}(x, t)+\mathcal{B} \tilde{Z}_{\tau}(x, t),
$$

where

$$
\mathcal{S}=\left(\begin{array}{lll}
D & 0 & 0 \\
0 & D & 0 \\
0 & 0 & D
\end{array}\right), \quad \mathcal{A}=\left(\begin{array}{ccc}
-(\alpha+\mu) & 0 & -S_{0} f^{\prime}(0) \\
\alpha & -(\mu+\beta) & -V_{0} g^{\prime}(0) \\
0 & 0 & -(\mu+\delta+\gamma)
\end{array}\right), \quad \mathcal{B}=\left(\begin{array}{lll}
0 & 0 & 0 \\
0 & 0 & 0 \\
0 & 0 & \rho
\end{array}\right),
$$


and $\rho=e^{-\mu \tau}\left(S_{0} f^{\prime}(0)+V_{0} g^{\prime}(0)\right)$.

Thus, we get

$$
\left(\lambda+\mu_{i} D+\mu+\alpha\right)\left(\lambda+\mu_{i} D+\mu+\beta\right)\left(\lambda+\mu_{i} D+\mu+\delta+\gamma-\rho e^{-\lambda \tau}\right)=0 .
$$

Clearly, (2.5) has eigenvalues $\lambda_{1}=-\left(\mu_{i} D+\mu+\alpha\right)<0$ and $\lambda_{2}=-\left(\mu_{i} D+\mu+\beta\right)<0$. The other eigenvalue $\lambda_{3}$ satisfies

$$
\lambda+\mu_{i} D+\mu+\delta+\gamma-\rho e^{-\lambda \tau}=0 .
$$

Define

$$
\lambda_{3}(\lambda, i)=-\mu_{i} D-(\mu+\delta+\gamma)\left(1-\mathcal{R}_{0} e^{-\lambda \tau}\right)=0 .
$$

If $\mathcal{R}_{0}<1$, then we have

$$
\lambda_{3}(0, i)=-\mu_{i} D-(\mu+\delta+\gamma)\left(1-\mathcal{R}_{0}\right)<0,
$$

and

$$
\frac{\partial \lambda_{3}(\lambda, i)}{\partial \lambda}=-(\mu+\delta+\gamma) \mathcal{R}_{0} \tau e^{-\lambda \tau}<0, \quad \forall \lambda \geq 0 .
$$

Thus, (2.6) has no positive real root.

Assume that (2.6) has a complex root $\lambda=\omega_{1}+\mathrm{i} \omega_{2}$ with $\omega_{1} \geq 0$; substituting it into (2.6), one has

$$
\begin{aligned}
& (\delta+\gamma+\mu) \mathcal{R}_{0} e^{-\omega_{1} \tau} \sin \omega_{2} \tau=-\omega_{2}, \\
& (\delta+\gamma+\mu) \mathcal{R}_{0} e^{-\omega_{1} \tau} \cos \omega_{2} \tau=\omega_{1}+\mu_{i} D+\mu+\delta+\gamma .
\end{aligned}
$$

Squaring and adding these equations together, we obtain

$$
(\mu+\delta+\gamma)^{2} \mathcal{R}_{0}^{2} e^{-2 \omega_{1} \tau}=\omega_{2}^{2}+\left(\omega_{1}+\mu_{i} D+\mu+\delta+\gamma\right)^{2} .
$$

Using $\omega_{1} \geq 0$ and $\mu_{i} \geq 0$, we have

$$
(\mu+\delta+\gamma)^{2} \mathcal{R}_{0}^{2} e^{-2 \omega_{1} \tau}<\omega_{2}^{2}+\left(\omega_{1}+\mu_{i} D+\mu+\delta+\gamma\right)^{2},
$$

when $\mathcal{R}_{0}<1$. This is a contradiction. Therefore, (2.6) has no complex root with a non-negative real part. Considering $i=0$ and the space $\mathbb{X}_{0}$ corresponding to $\mu_{0}=0$, we get

$$
\lambda_{3}(0,0)=-(\mu+\delta+\gamma)\left(1-\mathcal{R}_{0}\right)>0,
$$

and

$$
\lim _{\lambda \rightarrow+\infty} \lambda_{3}(\lambda, 0)=-(\mu+\delta+\gamma)<0,
$$

when $\mathcal{R}_{0}>1$. Therefore, there exists a constant $\lambda_{0}>0$ such that $\lambda_{3}\left(\lambda_{0}, 0\right)=0$, yielding that (2.6) has at least one positive root.

\subsection{Global stability}

Define $\Phi(x)=x-1-\ln x$. It is clear that $\Phi(x) \geq 0$ for all $x>0$. It follows from (H2) that $g^{\prime}(I)$ is nonincreasing, so one can obtain $g(I)=g(I)-g(0)=g^{\prime}(\eta)(I-0) \leq g^{\prime}(0) I$, where $\eta$ is between 0 and $I$. Similarly, one has $f(I) \leq f^{\prime}(0) I$.

Theorem 2.4 If $\mathcal{R}_{0} \leq 1$, then $E_{0}$ of system (2.1) is globally asymptotically stable. 
Proof Define

$$
L(t)=\int_{\Omega}\left(L_{1}(x, t)+L_{2}(x, t)\right) \mathrm{d} x
$$

where

$$
\begin{aligned}
& L_{1}(x, t)=e^{\mu \tau} I(x, t)+S_{0} \Phi\left(\frac{S(x, t)}{S_{0}}\right)+V_{0} \Phi\left(\frac{V(x, t)}{V_{0}}\right), \\
& L_{2}(x, t)=\int_{0}^{\tau}(f(I(x, t-\theta)) S(x, t-\theta)+g(I(x, t-\theta)) V(x, t-\theta)) \mathrm{d} \theta .
\end{aligned}
$$

According to $\ln x \leq x-1$ and

$$
\mu p A=(\mu+\alpha) S_{0}, \mu q A+\alpha S_{0}=(\mu+\beta) V_{0},
$$

we have

$$
\begin{aligned}
\frac{\partial L_{1}(x, t)}{\partial t}= & \left(1-\frac{S_{0}}{S}\right)\left(D \Delta S+(\alpha+\mu)\left(S_{0}-S\right)-S f(I)\right) \\
& +\left(1-\frac{V_{0}}{V}\right)\left(D \Delta V+\alpha\left(S-S_{0}\right)+(\mu+\beta)\left(V_{0}-V\right)-V g(I)\right) \\
& +e^{\mu \tau} D \Delta I+S_{\tau} f\left(I_{\tau}\right)+V_{\tau} g\left(I_{\tau}\right)-e^{\mu \tau}(\delta+\gamma+\mu) I, \\
\frac{\partial L_{2}(x, t)}{\partial t}= & S f(I)+V g(I)-S_{\tau} f\left(I_{\tau}\right)-V_{\tau} g\left(I_{\tau}\right),
\end{aligned}
$$

where $S_{\tau}=S(x, t-\tau), V_{\tau}=V(x, t-\tau)$ and $I_{\tau}=I(x, t-\tau)$.

Thus, we get

$$
\begin{aligned}
\frac{\partial\left(L_{1}+L_{2}\right)(x, t)}{\partial t}= & {\left[(\mu+\alpha) S_{0}\left(2-\frac{S_{0}}{S}-\frac{S}{S_{0}}\right)+(\mu+\beta) V_{0}\left(2-\frac{V_{0}}{V}-\frac{V}{V_{0}}\right)\right.} \\
& \left.+\alpha S_{0}\left(\frac{S}{S_{0}}+\frac{V_{0}}{V}-\frac{V_{0} S}{V S_{0}}-1\right)+S_{0} f(I)+V_{0} g(I)-e^{\mu \tau}(\mu+\delta+\gamma) I\right] \\
& +D\left(1-\frac{S_{0}}{S}\right) \Delta S+D\left(1-\frac{V_{0}}{V}\right) \Delta V+e^{\mu \tau} D \Delta I \\
\leq & -\left[(\mu+\alpha) S_{0}\left(\Phi\left(\frac{S_{0}}{S}\right)+\Phi\left(\frac{S}{S_{0}}\right)\right)+(\mu+\beta)\left(\Phi\left(\frac{V}{V_{0}}\right)+\Phi\left(\frac{V_{0}}{V}\right)\right) V_{0}\right. \\
& -\alpha S_{0}\left(\Phi\left(\frac{S}{S_{0}}\right)+\Phi\left(\frac{V_{0}}{V}\right)-\Phi\left(\frac{V_{0} S}{V S_{0}}\right)\right)-\left(S_{0} f^{\prime}(0)+V_{0} g^{\prime}(0)\right. \\
& \left.\left.-e^{\mu \tau}(\mu+\delta+\gamma)\right) I\right]+D\left(1-\frac{S_{0}}{S}\right) \Delta S+D\left(1-\frac{V_{0}}{V}\right) \Delta V+e^{\mu \tau} D \Delta I .
\end{aligned}
$$

Since

$$
\int_{\Omega} \Delta S \mathrm{~d} x=\int_{\Omega} \Delta V \mathrm{~d} x=0, \int_{\Omega} \frac{\Delta S}{S} \mathrm{~d} x=\int_{\Omega} \frac{|\nabla S|^{2}}{S^{2}} \mathrm{~d} x \geq 0, \int_{\Omega} \frac{\Delta V}{V} \mathrm{~d} x=\int_{\Omega} \frac{|\nabla V|^{2}}{V^{2}} \mathrm{~d} x \geq 0,
$$

we have

$$
\begin{aligned}
\frac{\mathrm{d} L(t)}{\mathrm{d} t}= & \int_{\Omega} \frac{\partial\left(L_{1}+L_{2}\right)(x, t)}{\partial t} \mathrm{~d} x \\
\leq & -\int_{\Omega}\left[(\mu+\alpha) S_{0} \Phi\left(\frac{S_{0}}{S}\right)+\mu S_{0} \Phi\left(\frac{S}{S_{0}}\right)+(\mu+\beta) V_{0} \Phi\left(\frac{V}{V_{0}}\right)+\mu q A \Phi\left(\frac{V_{0}}{V}\right)\right. \\
& \left.+\alpha S_{0} \Phi\left(\frac{V_{0} S}{V S_{0}}\right)+e^{\mu \tau}(\mu+\delta+\gamma)\left(1-\mathcal{R}_{0}\right) I\right] \mathrm{d} x \leq 0 .
\end{aligned}
$$

Clearly, the largest invariant subset of $\left\{\frac{\mathrm{d} L(t)}{\mathrm{d} t}=0\right\}$ is $\left\{E_{0}\right\}$. The conclusion is correct. 
Theorem 2.5 If $\mathcal{R}_{0}>1$, then $E^{*}$ of system (2.1) is globally asymptotically stable.

Proof Define

$$
H(t)=\int_{\Omega}\left(H_{1}(x, t)+H_{2}(x, t)\right) \mathrm{d} x
$$

where

$$
\begin{aligned}
& H_{1}(x, t)=S^{*} \Phi\left(\frac{S}{S^{*}}\right)+V^{*} \Phi\left(\frac{V}{V^{*}}\right)+e^{\mu \tau} I^{*} \Phi\left(\frac{I}{I^{*}}\right), \\
& H_{2}(x, t)=S^{*} f\left(I^{*}\right) \int_{0}^{\tau} \Phi\left(\frac{S_{\theta} f\left(I_{\theta}\right)}{S^{*} f\left(I^{*}\right)}\right) \mathrm{d} \theta+V^{*} g\left(I^{*}\right) \int_{0}^{\tau} \Phi\left(\frac{V_{\theta} g\left(I_{\theta}\right)}{V^{*} g\left(I^{*}\right)}\right) \mathrm{d} \theta .
\end{aligned}
$$

Clearly, $H \geq 0$ with the equality holds if and only if $S=S^{*}, V=V^{*}$ and $I=I^{*}$.

It follows from (2.4) that

$$
\begin{aligned}
\frac{\partial H_{1}(x, t)}{\partial t}= & \left(1-\frac{S^{*}}{S}\right)\left(D \Delta S+(\alpha+\mu) S^{*}+S^{*} f\left(I^{*}\right)-S f(I)-(\alpha+\mu) S\right) \\
& +\left(1-\frac{V^{*}}{V}\right)\left(D \triangle V+\alpha\left(S-S^{*}\right)+(\mu+\beta)\left(V^{*}-V\right)+g\left(I^{*}\right) V^{*}-g(I) V\right) \\
& +\left(1-\frac{I^{*}}{I}\right)\left(D \Delta I+S_{\tau} f\left(I_{\tau}\right)+V_{\tau} g\left(I_{\tau}\right)-e^{\mu \tau}(\mu+\delta+\gamma) I\right), \\
\frac{\partial H_{2}(x, t)}{\partial t}= & S f(I)-S_{\tau} f\left(I_{\tau}\right)+S^{*} f\left(I^{*}\right) \ln \left(\frac{S_{\tau} f\left(I_{\tau}\right)}{S f(I)}\right) \\
& +g(I) V-g\left(I_{\tau}\right) V_{\tau}+g\left(I^{*}\right) V^{*} \ln \left(\frac{g\left(I_{\tau}\right) V_{\tau}}{g(I) V}\right) .
\end{aligned}
$$

Notice that

$$
\int_{\Omega} \Delta S \mathrm{~d} x=\int_{\Omega} \Delta V \mathrm{~d} x=\int_{\Omega} \Delta I \mathrm{~d} x=0
$$

and

$$
\int_{\Omega} \frac{\Delta S}{S} \mathrm{~d} x=\int_{\Omega} \frac{|\nabla S|^{2}}{S^{2}} \mathrm{~d} x \geq 0, \int_{\Omega} \frac{\Delta V}{V} \mathrm{~d} x=\int_{\Omega} \frac{|\nabla V|^{2}}{V^{2}} \mathrm{~d} x \geq 0, \int_{\Omega} \frac{\Delta I}{I} \mathrm{~d} x=\int_{\Omega} \frac{|\nabla I|^{2}}{I^{2}} \mathrm{~d} x \geq 0,
$$

SO

$$
\begin{aligned}
\frac{\mathrm{d} H(t)}{\mathrm{d} t}= & \int_{\Omega} \frac{\partial\left(H_{1}(x, t)+H_{2}(x, t)\right)}{\partial t} \mathrm{~d} x \\
\leq & \int_{\Omega}\left[(\mu+\alpha) S^{*}\left(2-\frac{S^{*}}{S}-\frac{S}{S^{*}}\right)+(\mu+\beta) V^{*}\left(2-\frac{V^{*}}{V}-\frac{V}{V^{*}}\right)\right. \\
& +\alpha S^{*}\left(\frac{S}{S^{*}}+\frac{V^{*}}{V}-\frac{S V^{*}}{S^{*} V}-1\right) \\
& +S^{*} f\left(I^{*}\right)\left(2-\frac{S^{*}}{S}+\frac{f(I)}{f\left(I^{*}\right)}-\frac{I^{*} S_{\tau} f\left(I_{\tau}\right)}{I S^{*} f\left(I^{*}\right)}-\frac{I}{I^{*}}+\ln \left(\frac{S_{\tau} f\left(I_{\tau}\right)}{S f(I)}\right)\right) \\
& \left.+V^{*} g\left(I^{*}\right)\left(2-\frac{V^{*}}{V}+\frac{g(I)}{g\left(I^{*}\right)}-\frac{I^{*} V_{\tau} g\left(I_{\tau}\right)}{I V^{*} g\left(I^{*}\right)}-\frac{I}{I^{*}}+\ln \left(\frac{V_{\tau} g\left(I_{\tau}\right)}{V g(I)}\right)\right)\right] d x \\
= & -\int_{\Omega}\left[\mu S^{*}\left(\Phi\left(\frac{S^{*}}{S}\right)+\Phi\left(\frac{S}{S^{*}}\right)\right)+\alpha S^{*}\left(\Phi\left(\frac{S^{*}}{S}\right)+\Phi\left(\frac{S V^{*}}{S^{*} V}\right)\right)\right. \\
& +\mu q A \Phi\left(\frac{V^{*}}{V}\right)+(\mu+\beta) V^{*} \Phi\left(\frac{V}{V^{*}}\right) \\
& +S^{*} f\left(I^{*}\right)\left(\Phi\left(\frac{I^{*} S_{\tau} f\left(I_{\tau}\right)}{I S^{*} f\left(I^{*}\right)}\right)+\Phi\left(\frac{S^{*}}{S}\right)-\frac{f(I)}{f\left(I^{*}\right)}+\frac{I}{I^{*}}-\ln \left(\frac{I f\left(I^{*}\right)}{I^{*} f(I)}\right)\right)
\end{aligned}
$$




$$
\left.+V^{*} g\left(I^{*}\right)\left(\Phi\left(\frac{I^{*} V_{\tau} g\left(I_{\tau}\right)}{I V^{*} g\left(I^{*}\right)}\right)-\frac{g(I)}{g\left(I^{*}\right)}+\frac{I}{I^{*}}-\ln \left(\frac{I g\left(I^{*}\right)}{I^{*} g\left(I^{*}\right)}\right)\right)\right] \mathrm{d} x .
$$

By Assumption (H2) and $\ln x \leq x-1$, we can get

$$
\ln \left(\frac{I \mathcal{G}\left(I^{*}\right)}{I^{*} \mathcal{G}(I)}\right)+\frac{\mathcal{G}(I)}{\mathcal{G}\left(I^{*}\right)}-\frac{I}{I^{*}} \leq \frac{I \mathcal{G}\left(I^{*}\right)}{I^{*} \mathcal{G}(I)}+\frac{\mathcal{G}(I)}{\mathcal{G}\left(I^{*}\right)}-\frac{I}{I^{*}}-1 \leq 0
$$

where $\mathcal{G}=\{f, g\}$. Hence,

$$
\begin{aligned}
\frac{\mathrm{d} H(t)}{\mathrm{d} t}= & \int_{\Omega} \frac{\partial\left(H_{1}(x, t)+H_{2}(x, t)\right)}{\partial t} \mathrm{~d} x \\
\leq & -\int_{\Omega}\left[\mu S^{*}\left(\Phi\left(\frac{S^{*}}{S}\right)+\Phi\left(\frac{S}{S^{*}}\right)\right)+\alpha S^{*}\left(\Phi\left(\frac{S^{*}}{S}\right)+\Phi\left(\frac{S V^{*}}{S^{*} V}\right)\right)\right. \\
& +\mu q A \Phi\left(\frac{V^{*}}{V}\right)+(\mu+\beta) V^{*} \Phi\left(\frac{V}{V^{*}}\right) \\
& \left.+S^{*} f\left(I^{*}\right)\left(\Phi\left(\frac{S^{*}}{S}\right)+\Phi\left(\frac{I^{*} S_{\tau} f\left(I_{\tau}\right)}{I S^{*} f\left(I^{*}\right)}\right)\right)+V^{*} g\left(I^{*}\right) \Phi\left(\frac{I^{*} V_{\tau} g\left(I_{\tau}\right)}{I V^{*} g\left(I^{*}\right)}\right)\right] \mathrm{d} x \\
\leq & 0 .
\end{aligned}
$$

In a manner similar to the proof of Theorem 2.4, the conclusion is proved.

\section{A Discretized Model}

Define $\Omega=[a, b], \triangle x=(b-a) / M$ and $m=[\tau / \Delta t] . \Delta t$ is the time stepsize. The mesh points are $\left(x_{n}, t_{k}\right)$, where $x_{n}=a+n \triangle x$ and $t_{k}=k \Delta t$ with $n \in\{0,1, \ldots, M\}$ and $k \in \mathbb{N}$. Denote $S\left(x_{n}, t_{k}\right), V\left(x_{n}, t_{k}\right)$ and $I\left(x_{n}, t_{k}\right)$ by $S_{n}^{k}, V_{n}^{k}$ and $I_{n}^{k}$, respectively. We use a $(M+1)$-dimensional vector

$$
U^{k}=\left(U_{0}^{k}, U_{1}^{k}, \ldots, U_{M}^{k}\right)^{T}
$$

to denote the approximation $S, V$ and $I$ at time $t_{k} .()^{T}$ is the transposition of a vector. According to the NSFD scheme, the discretization of system (2.1) is

$$
\left\{\begin{aligned}
\frac{S_{n}^{k+1}-S_{n}^{k}}{\Delta t}= & D_{1} \frac{S_{n+1}^{k+1}-2 S_{n}^{k+1}+S_{n-1}^{k+1}}{(\triangle x)^{2}}+\mu p A-S_{n}^{k+1} f\left(I_{n}^{k}\right)-(\mu+\alpha) S_{n}^{k+1}, \\
\frac{V_{n}^{k+1}-V_{n}^{k}}{\Delta t}= & D_{2} \frac{V_{n+1}^{k+1}-2 V_{n}^{k+1}+V_{n-1}^{k+1}}{(\triangle x)^{2}}+\mu q A+\alpha S_{n}^{k+1}-V_{n}^{k+1} g\left(I_{n}^{k}\right)-(\mu+\beta) V_{n}^{k+1}, \\
\frac{I_{n}^{k+1}-I_{n}^{k}}{\Delta t}= & D_{4} \frac{I_{n+1}^{k+1}-2 I_{n}^{k+1}+I_{n-1}^{k+1}}{(\triangle x)^{2}} \\
& +e^{-\mu \tau}\left[S_{n}^{k+1-m} f\left(I_{n}^{k-m}\right)+V_{n}^{k+1-m} g\left(I_{n}^{k-m}\right)\right]-(\mu+\delta+\gamma) I_{n}^{k+1},
\end{aligned}\right.
$$

with initial condition

$$
S_{n}^{k}=\phi_{n}^{k} \geq 0, \quad V_{n}^{k}=\psi_{n}^{k} \geq 0 \text { and } \quad I_{n}^{0}=\varphi_{n}^{k} \geq 0,
$$

where $k \in\{-m,-m+1, \ldots, 0\}$ and $n \in\{0,1, \ldots, M\}$ and the boundary condition is

$$
S_{-1}^{k}=S_{0}^{k}, S_{M}^{k}=S_{M+1}^{k}, V_{-1}^{k}=V_{0}^{k}, V_{M}^{k}=V_{M+1}^{k}, I_{-1}^{k}=I_{0}^{k}, I_{M}^{k}=I_{M+1}^{k}, k \in \mathbb{N} .
$$

The equilibria of system (3.1) is the same as for (2.1). Applying M-matrix theory [39], we have the following result: 
Theorem 3.1 For any $\Delta x>0$ and $\Delta t>0$, the solution of system (3.1) with (3.2) and (3.3) is nonnegative and bounded.

Proof According to (3.1), we get

$$
\mathcal{A}^{k} S^{k+1}=S^{k}+\mu p A \Delta t \mathbf{e},
$$

where

$$
\mathcal{A}^{k}=\left(\begin{array}{ccccccc}
a_{0}^{k} & a & 0 & \ldots & 0 & 0 & 0 \\
a & a_{1}^{k} & a & \ldots & 0 & 0 & 0 \\
0 & a & a_{2}^{k} & \ldots & 0 & 0 & 0 \\
\vdots & \vdots & \vdots & \ddots & \vdots & \vdots & \vdots \\
0 & 0 & 0 & \ldots & a_{M-2}^{k} & a & 0 \\
0 & 0 & 0 & \ldots & a & a_{M-1}^{k} & a \\
0 & 0 & 0 & \ldots & 0 & a & a_{M}^{k}
\end{array}\right)
$$

and $\mathbf{e}=(1,1, \ldots, 1)^{T}$. We have the coefficients $a=-D_{1} \Delta t /(\triangle x)^{2}, a_{0}^{k}=1+D_{1} \Delta t /(\triangle x)^{2}+$ $\Delta t\left(f\left(I_{0}^{k}\right)+\mu+\alpha\right), a_{M}^{k}=1+D_{1} \Delta t /(\Delta x)^{2}+\Delta t\left(f\left(I_{M}^{k}\right)+\mu+\alpha\right)$ and $a_{i}^{k}=1+2 D_{1} \Delta t /(\Delta x)^{2}+$ $\Delta t\left(f\left(I_{i}^{k}\right)+\mu+\alpha\right)(i=1,2, \ldots, M-1)$. Since $\mathcal{A}^{k}$ is a strictly diagonally dominant matrix, we can obtain

$$
S^{k+1}=\left(\mathcal{A}^{k}\right)^{-1}\left(S^{k}+\mu p A \Delta t \mathbf{e}\right)>0 .
$$

From (3.1) again, one has

$$
\mathcal{B}^{k} V^{k+1}=V^{k}+\left(\mu q A \mathbf{e}+\alpha S^{k+1}\right) \Delta t
$$

where

$$
\mathcal{B}^{k}=\left(\begin{array}{ccccccc}
b_{0}^{k} & b & 0 & \ldots & 0 & 0 & 0 \\
b & b_{1}^{k} & b & \ldots & 0 & 0 & 0 \\
0 & b & b_{2}^{k} & \ldots & 0 & 0 & 0 \\
\vdots & \vdots & \vdots & \ddots & \vdots & \vdots & \vdots \\
0 & 0 & 0 & \ldots & b_{M-2}^{k} & b & 0 \\
0 & 0 & 0 & \ldots & b & b_{M-1}^{k} & b \\
0 & 0 & 0 & \ldots & 0 & b & b_{M}^{k}
\end{array}\right),
$$

with $b=-D_{2} \Delta t /(\Delta x)^{2}, b_{0}^{k}=1+D_{2} \Delta t /(\Delta x)^{2}+\Delta t\left(g\left(I_{0}^{k}\right)+\mu+\beta\right), b_{M}^{k}=1+D_{2} \Delta t /(\Delta x)^{2}+$ $\Delta t\left(g\left(I_{M}^{k}\right)+\mu+\beta\right)$, and $b_{i}^{k}=1+2 D_{2} \Delta t /(\Delta x)^{2}+\Delta t\left(g\left(I_{i}^{k}\right)+\mu+\beta\right)(i=1,2, \ldots, M-1)$. Since $B^{k}$ is a M-matrix, we get

$$
V^{k+1}=\left(\mathcal{B}^{k}\right)^{-1}\left(V^{k}+\left(\mu q A \mathbf{e}+\alpha S^{k+1}\right) \Delta t\right) .
$$

Similarly, we also have

$$
\mathcal{C} I^{k+1}=e^{-\mu \tau} \Delta t T^{k+1}+I^{k} .
$$

Here $T^{k+1}=\left(S_{0}^{k+1-m} f\left(I_{0}^{k-m}\right)+V_{0}^{k+1-m} g\left(I_{0}^{k-m}\right), \ldots, S_{M}^{k+1-m} f\left(I_{M}^{k-m}\right)+V_{M}^{k+1-m} g\left(I_{M}^{k-m}\right)\right)^{T}$ 
and

$$
\mathcal{C}=\left(\begin{array}{ccccccc}
c_{1} & c_{2} & 0 & \ldots & 0 & 0 & 0 \\
c_{2} & c_{3} & c_{2} & \ldots & 0 & 0 & 0 \\
0 & c_{2} & c_{3} & \ldots & 0 & 0 & 0 \\
\vdots & \vdots & \vdots & \ddots & \vdots & \vdots & \vdots \\
0 & 0 & 0 & \ldots & c_{3} & c_{2} & 0 \\
0 & 0 & 0 & \ldots & c_{2} & c_{3} & c_{2} \\
0 & 0 & 0 & \ldots & 0 & c_{2} & c_{1}
\end{array}\right)
$$

with $c_{1}=1+D_{4} \Delta t /(\Delta x)^{2}+\Delta t(\mu+\delta+\gamma), c_{2}=-D_{4} \Delta t /(\Delta x)^{2}$ and $c_{3}=1+2 D_{4} \Delta t /(\Delta x)^{2}+$ $\Delta t(\mu+\delta+\gamma)$. Since $\mathcal{C}$ is a M-matrix, one has

$$
I^{k+1}=\mathcal{C}^{-1}\left(e^{-\mu \tau} \Delta t T^{k+1}+I^{k}\right) .
$$

Thus, the solution of system (3.1) remains nonnegative.

Define

$$
G^{k}=\sum_{n=0}^{M}\left(S_{n}^{k}+V_{n}^{k}+e^{\mu \tau} I_{n}^{k+m}\right) .
$$

By (3.1), we get

$$
\begin{aligned}
\frac{G^{k+1}-G^{k}}{\Delta t} & =\mu A(M+1)-\sum_{n=0}^{M}\left(\mu S_{n}^{k+1}+(\mu+\beta) V_{n}^{k+1}+e^{\mu \tau}(\mu+\delta+\gamma) I_{n}^{k+m+1}\right) \\
& <\mu A(M+1)-\mu G^{k+1} .
\end{aligned}
$$

Thus,

$$
G^{k+1} \leq \frac{\mu A(M+1) \Delta t+G^{k}}{1+\mu \Delta t}
$$

It can be concluded by induction that $\limsup _{k \rightarrow+\infty} G^{k} \leq(M+1) A$. Therefore, for all $k \in \mathbb{N}$, $\left\{S^{k}\right\},\left\{V^{k}\right\},\left\{I^{k}\right\}$ are bounded.

\section{Global Stability of the Discretized System (3.1)}

In this section, we discuss the global stability of equilibria for system (3.1).

Theorem 4.1 For any $\Delta x>0$ and $\Delta t>0$, if $\mathcal{R}_{0} \leq 1$, then $E_{0}$ of system (3.1) is globally asymptotically stable.

Proof Define $L^{k}=L_{1}^{k}+L_{2}^{k}$, where

$$
L_{1}^{k}=\frac{1}{\Delta t} \sum_{n=0}^{M}\left[S_{0} \Phi\left(\frac{S_{n}^{k}}{S_{0}}\right)+V_{0} \Phi\left(\frac{V_{n}^{k}}{V_{0}}\right)+e^{\mu \tau}(1+(\mu+\delta+\gamma) \Delta t) I_{n}^{k}\right]
$$

and

$$
L_{2}^{k}=\sum_{n=0}^{M} \sum_{j=k-m}^{k-1}\left(S_{n}^{j+1} f\left(I_{n}^{j}\right)+V_{n}^{j+1} g\left(I_{n}^{j}\right)\right) .
$$

Clearly, $L^{k} \geq 0$ with equality holds if and only if $S_{n}^{k}=0, V_{n}^{k}=0$ and $I_{n}^{k}=0$ for all $k \in \mathbb{N}$ and $n \in\{1,2, \ldots, M\}$. 
Applying $\mu p A=(\mu+\alpha) S_{0}, \mu q A+\alpha S_{0}=(\mu+\beta) V_{0}$, we can get

$$
\begin{aligned}
L_{1}^{k+1}-L_{1}^{k} \leq & \frac{1}{\Delta t} \sum_{n=0}^{M}\left[\left(1-\frac{S_{0}}{S_{n}^{k+1}}\right)\left(S_{n}^{k+1}-S_{n}^{k}\right)+\left(1-\frac{V_{0}}{V_{n}^{k+1}}\right)\left(V_{n}^{k+1}-V_{n}^{k}\right)\right. \\
& \left.+e^{\mu \tau}(1+(\mu+\delta+\gamma) \Delta t)\left(I_{n}^{k+1}-I_{n}^{k}\right)\right] \\
= & \sum_{n=0}^{M}\left[\left(1-\frac{S_{0}}{S_{n}^{k+1}}\right)\left((\mu+\alpha)\left(S_{0}-S_{n}^{k+1}\right)-S_{n}^{k+1} f\left(I_{n}^{k}\right)\right)\right. \\
& +\left(1-\frac{V_{0}}{V_{n}^{k+1}}\right)\left(\alpha\left(S_{n}^{k+1}-S_{0}\right)+(\mu+\beta)\left(V_{0}-V_{n}^{k+1}\right)-V_{n}^{k+1} g\left(I_{n}^{k}\right)\right) \\
& +S_{n}^{k+1-m} f\left(I_{n}^{k-m}\right)+V_{n}^{k+1-m} g\left(I_{n}^{k-m}\right)-e^{\mu \tau}(\mu+\delta+\gamma) I_{n}^{k+1} \\
& \left.+e^{\mu \tau}(\mu+\delta+\gamma)\left(I_{n}^{k+1}-I_{n}^{k}\right)\right]+\Delta \bar{L}^{k},
\end{aligned}
$$

where

$$
\begin{aligned}
\Delta \bar{L}^{k}= & \sum_{n=0}^{M} \frac{1}{(\Delta x)^{2}}\left[D_{1}\left(1-\frac{S_{0}}{S_{n}^{k+1}}\right)\left(S_{n+1}^{k+1}-2 S_{n}^{k+1}+S_{n-1}^{k+1}\right)\right. \\
& +D_{2}\left(1-\frac{V_{0}}{V_{n}^{k+1}}\right)\left(V_{n+1}^{k+1}-2 V_{n}^{k+1}+V_{n-1}^{k+1}\right) \\
& \left.+e^{\mu \tau} D_{4}\left(I_{n+1}^{k+1}-2 I_{n}^{k+1}+I_{n-1}^{k+1}\right)\right]
\end{aligned}
$$

and

$$
L_{2}^{k+1}-L_{2}^{k}=\sum_{n=0}^{M}\left(S_{n}^{k+1} f\left(I_{n}^{k}\right)+V_{n}^{k+1} g\left(I_{n}^{k}\right)-S_{n}^{k+1-m} f\left(I_{n}^{k-m}\right)-V_{n}^{k+1-m} g\left(I_{n}^{k-m}\right)\right) .
$$

For $n \in\{0,1, \ldots, M-1\}$, we have

$$
\frac{S_{n+1}^{k+1}}{S_{n}^{k+1}}-2+\frac{S_{n}^{k+1}}{S_{n+1}^{k+1}} \geq 0, \quad \frac{V_{n+1}^{k+1}}{V_{n}^{k+1}}-2+\frac{V_{n}^{k+1}}{V_{n+1}^{k+1}} \geq 0 .
$$

Then,

$$
\begin{aligned}
\Delta \bar{L}^{k}= & \sum_{n=0}^{M} \frac{1}{(\Delta x)^{2}}\left[D_{1}\left(S_{n+1}^{k+1}-2 S_{n}^{k+1}+S_{n-1}^{k+1}\right)-S_{0} D_{1}\left(\frac{S_{n+1}^{k+1}}{S_{n}^{k+1}}-2+\frac{S_{n-1}^{k+1}}{S_{n}^{k+1}}\right)\right. \\
& +D_{2}\left(V_{n+1}^{k+1}-2 V_{n}^{k+1}+V_{n-1}^{k+1}\right)-V_{0} D_{2}\left(\frac{V_{n+1}^{k+1}}{V_{n}^{k+1}}-2+\frac{V_{n-1}^{k+1}}{V_{n}^{k+1}}\right) \\
& \left.+e^{\mu \tau} D_{4}\left(I_{n+1}^{k+1}-2 I_{n}^{k+1}+I_{n-1}^{k+1}\right)\right] \\
\leq & \frac{1}{(\Delta x)^{2}}\left[D_{1}\left(S_{M+1}^{k+1}-S_{M}^{k+1}+S_{-1}^{k+1}-S_{0}^{k+1}\right)-S_{0} D_{1}\left(\frac{S_{-1}^{k+1}}{S_{0}^{k+1}}-2+\frac{S_{M+1}^{k+1}}{S_{M}^{k+1}}\right)\right. \\
& +D_{2}\left(V_{M+1}^{k+1}-V_{M}^{k+1}+V_{-1}^{k+1}-V_{0}^{k+1}\right)-V_{0} D_{2}\left(\frac{V_{-1}^{k+1}}{V_{0}^{k+1}}-2+\frac{V_{M+1}^{k+1}}{V_{M}^{k+1}}\right) \\
& \left.+e^{\mu \tau} D_{4}\left(I_{M+1}^{k+1}-I_{M}^{k+1}+I_{-1}^{k+1}-I_{0}^{k+1}\right)\right]=0 .
\end{aligned}
$$


Thus,

$$
\begin{aligned}
& L^{k+1}-L^{k} \leq \sum_{n=0}^{M}\left[(\mu+\alpha) S_{0}\left(2-\frac{S_{0}}{S_{n}^{k+1}}-\frac{S_{n}^{k+1}}{S_{0}}\right)+(\mu+\beta) V_{0}\left(2-\frac{V_{0}}{V_{n}^{k+1}}-\frac{V_{n}^{k+1}}{V_{0}}\right)\right. \\
& +\alpha S_{0}\left(\frac{S_{n}^{k+1}}{S_{0}}+\frac{V_{0}}{V_{n}^{k+1}}-\frac{V_{0} S_{n}^{k+1}}{V_{n}^{k+1} S_{0}}-1\right)+S_{0} f\left(I_{n}^{k}\right)+V_{0} g\left(I_{n}^{k}\right) \\
& \left.-e^{\mu \tau}(\mu+\delta+\gamma) I_{n}^{k}\right] \\
& \leq-\sum_{n=0}^{M}\left[(\mu+\alpha) S_{0}\left(\Phi\left(\frac{S_{0}}{S_{n}^{k+1}}\right)+\Phi\left(\frac{S_{n}^{k+1}}{S_{0}}\right)\right)+(\mu+\beta) V_{0}\left(\Phi\left(\frac{V_{0}}{V_{n}^{k+1}}\right)\right.\right. \\
& \left.+\Phi\left(\frac{V_{n}^{k+1}}{V_{0}}\right)\right)-\alpha S_{0}\left(\Phi\left(\frac{S_{n}^{k+1}}{S_{0}}\right)+\Phi\left(\frac{V_{0}}{V_{n}^{k+1}}\right)-\Phi\left(\frac{V_{0} S_{n}^{k+1}}{V_{n}^{k+1} S_{0}}\right)\right) \\
& \left.-\left(S_{0} f^{\prime}(0)+V_{0} g^{\prime}(0)-e^{\mu \tau}(\mu+\delta+\gamma)\right) I_{n}^{k}\right] \\
& =-\sum_{n=0}^{M}\left[(\mu+\alpha) S_{0} \Phi\left(\frac{S_{0}}{S_{n}^{k+1}}\right)+\mu S_{0} \Phi\left(\frac{S_{n}^{k+1}}{S_{0}}\right)+(\mu+\beta) V_{0} \Phi\left(\frac{V_{n}^{k+1}}{V_{0}}\right)\right. \\
& \left.\left.+\mu q A \Phi\left(\frac{V_{0}}{V_{n}^{k+1}}\right)+\alpha S_{0} \Phi\left(\frac{V_{0} S_{n}^{k+1}}{V_{n}^{k+1} S_{0}}\right)\right)+e^{\mu \tau}(\mu+\delta+\gamma)\left(1-\mathcal{R}_{0}\right) I_{n}^{k}\right] \text {. }
\end{aligned}
$$

It is clear that $L^{k+1}-L^{k} \leq 0$, when $\mathcal{R}_{0} \leq 1$. Then $\left\{L^{k}\right\}$ is a non-increasing sequence. There must exist $\tilde{L}>0$ such that $\lim _{k \rightarrow+\infty} L^{k}=\tilde{L}$, meaning that $\lim _{k \rightarrow+\infty}\left(L^{k+1}-L^{k}\right)=0$. Thus, we have

$$
\lim _{k \rightarrow+\infty} S_{n}^{k}=S_{0} \text { and } \lim _{k \rightarrow+\infty} V_{n}^{k}=V_{0} .
$$

Furthermore, we can get $\lim _{k \rightarrow+\infty} I_{n}^{k}=0$.

Theorem 4.2 For any $\Delta x>0$ and $\Delta t>0$, if $\mathcal{R}_{0}>1$, then $E^{*}$ of system (3.1) is globally asymptotically stable.

Proof Define $H^{k}=H_{1}^{k}+H_{2}^{k}$, where

$$
H_{1}^{k}=\frac{1}{\Delta t} \sum_{n=0}^{M}\left[S^{*} \Phi\left(\frac{S_{n}^{k}}{S^{*}}\right)+V^{*} \Phi\left(\frac{V_{n}^{k}}{V^{*}}\right)+e^{\mu \tau} I^{*} \Phi\left(\frac{I_{n}^{k}}{I^{*}}\right)\right]
$$

and

$$
\begin{aligned}
H_{2}^{k}= & \sum_{n=0}^{M} \sum_{j=k-m}^{k-1}\left[S^{*} f\left(I^{*}\right) \Phi\left(\frac{S_{n}^{j+1} f\left(I_{n}^{j}\right)}{S^{*} f\left(I^{*}\right)}\right)+V^{*} g\left(I^{*}\right) \Phi\left(\frac{V_{n}^{j+1} g\left(I_{n}^{j}\right)}{V^{*} g\left(I^{*}\right)}\right)\right. \\
& \left.+\left(f\left(I^{*}\right) S^{*}+g\left(I^{*}\right) V^{*}\right) \Phi\left(\frac{I_{n}^{k}}{I^{*}}\right)\right] .
\end{aligned}
$$

We conclude that $H^{k} \geq 0$ if and if only $S_{n}^{k}=S^{*}, V_{n}^{k}=V^{*}$ and $I_{n}^{k}=I^{*}$ for all $k \in \mathbb{N}$ and $n \in\{0,1, \ldots, M\}$.

By (2.4), we obtain

$$
\begin{aligned}
H_{1}^{k+1}-H_{1}^{k} \leq & \sum_{n=0}^{M} \frac{1}{\Delta t}\left[\left(1-\frac{S^{*}}{S_{n}^{k+1}}\right)\left(S_{n}^{k+1}-S_{n}^{k}\right)+\left(1-\frac{V^{*}}{V_{n}^{k+1}}\right)\left(V_{n}^{k+1}-V_{n}^{k}\right)\right. \\
& +e^{\mu \tau}\left(1-\frac{I^{*}}{I_{n}^{k+1}}\right)\left(I_{n}^{k+1}-I_{n}^{k}\right)
\end{aligned}
$$




$$
\begin{aligned}
= & \sum_{n=0}^{M}\left[\left(1-\frac{S^{*}}{S_{n}^{k+1}}\right)\left((\mu+\alpha) S^{*}+S^{*} f\left(I^{*}\right)-S_{n}^{k+1} f\left(I_{n}^{k}\right)-(\mu+\alpha) S_{n}^{k+1}\right)\right. \\
& +\left(1-\frac{V^{*}}{V_{n}^{k+1}}\right)\left(\alpha S_{n}^{k+1}-\alpha S^{*}+(\mu+\beta)\left(V^{*}-V_{n}^{k+1}\right)+V^{*} g\left(I^{*}\right)-V_{n}^{k+1} g\left(I_{n}^{k}\right)\right) \\
& \left.\left(1-\frac{I^{*}}{I_{n}^{k+1}}\right)\left(f\left(I_{n}^{k-m}\right) S_{n}^{k+1-m}+g\left(I_{n}^{k-m}\right) V_{n}^{k+1-m}-e^{\mu \tau}(\mu+\delta+\gamma) I_{n}^{k+1}\right)\right]+\Delta \bar{H}^{k} \\
= & \sum_{n=0}^{M}\left[(\mu+\alpha) S^{*}\left(2-\frac{S^{*}}{S_{n}^{k+1}}-\frac{S_{n}^{k+1}}{S^{*}}\right)+(\mu+\beta) V^{*}\left(2-\frac{V^{*}}{V_{n}^{k+1}}-\frac{V_{n}^{k+1}}{V^{*}}\right)\right. \\
& +\alpha S^{*}\left(\frac{S_{n}^{k+1}}{S^{*}}+\frac{V^{*}}{V_{n}^{k+1}}-\frac{S_{n}^{k+1} V^{*}}{S^{*} V_{n}^{k+1}}-1\right)+S_{n}^{k+1-m} f\left(I_{n}^{k-m}\right)+V_{n}^{k+1-m} g\left(I_{n}^{k-m}\right) \\
& -S_{n}^{k+1} f\left(I_{n}^{k}\right)-V_{n}^{k+1} g\left(V_{n}^{k}\right) \\
& +S^{*} f\left(I^{*}\right)\left(2-\frac{S^{*}}{S_{n}^{k+1}}+\frac{f\left(I_{n}^{k}\right)}{f\left(I^{*}\right)}-\frac{I^{*} S_{n}^{k+1-m} f\left(I_{n}^{k-m}\right)}{I_{n}^{k+1} S^{*} f\left(I^{*}\right)}-\frac{I_{n}^{k+1}}{I^{*}}\right) \\
& \left.+V^{*} g\left(I^{*}\right)\left(2-\frac{V^{*}}{V_{n}^{k+1}}+\frac{g\left(I_{n}^{k}\right)}{g\left(I^{*}\right)}-\frac{I^{*} V_{n}^{k+1-m} g\left(I_{n}^{k-m}\right)}{I_{n}^{k+1} V^{*} g\left(I^{*}\right)}-\frac{I_{n}^{k+1}}{I^{*}}\right)\right]+\Delta \bar{H}^{k}
\end{aligned}
$$

where

$$
\begin{aligned}
\Delta \bar{H}^{k}= & \frac{1}{(\Delta x)^{2}} \sum_{n=0}^{M}\left[D_{1}\left(1-\frac{S^{*}}{S_{n}^{k+1}}\right)\left(S_{n+1}^{k+1}-2 S_{n}^{k+1}+S_{n-1}^{k+1}\right)\right. \\
& +D_{2}\left(1-\frac{V^{*}}{V_{n}^{k+1}}\right)\left(V_{n+1}^{k+1}-2 V_{n}^{k+1}+V_{n-1}^{k+1}\right) \\
& +e^{\mu \tau} D_{4}\left(1-\frac{I^{*}}{I_{n}^{k+1}}\right)\left(I_{n+1}^{k+1}-2 I_{n}^{k+1}+I_{n-1}^{k+1}\right) \\
= & \frac{1}{(\Delta x)^{2}} \sum_{n=0}^{M}\left[D_{1}\left(S_{n+1}^{k+1}-2 S_{n}^{k+1}+S_{n-1}^{k+1}\right)-S^{*} D_{1}\left(\frac{S_{n+1}^{k+1}}{S_{n}^{k+1}}-2+\frac{S_{n-1}^{k+1}}{S_{n}^{k+1}}\right)\right. \\
& +D_{2}\left(V_{n+1}^{k+1}-2 V_{n}^{k+1}+V_{n-1}^{k+1}\right)-V^{*} D_{2}\left(\frac{V_{n+1}^{k+1}}{V_{n}^{k+1}}-2+\frac{V_{n-1}^{k+1}}{V_{n}^{k+1}}\right) \\
& \left.+e^{\mu \tau} D_{4}\left(I_{n+1}^{k+1}-2 I_{n}^{k+1}+I_{n-1}^{k+1}\right)-I^{*} D_{4}\left(\frac{I_{n+1}^{k+1}}{I_{n}^{k+1}}-2+\frac{I_{n-1}^{k+1}}{I_{n}^{k+1}}\right)\right]
\end{aligned}
$$

and

$$
\begin{aligned}
H_{2}^{k+1}-H_{2}^{k}= & \sum_{n=0}^{M}\left[S_{n}^{k+1} f\left(I_{n}^{k}\right)-S_{n}^{k+1-m} f\left(I_{n}^{k-m}\right)+S^{*} f\left(I^{*}\right) \ln \frac{S_{n}^{k+1-m} f\left(I_{n}^{k-m}\right)}{S_{n}^{k+1} f\left(I_{n}^{k}\right)}\right. \\
& +V_{n}^{k+1} g\left(V_{n}^{k}\right)-V_{n}^{k+1-m} g\left(I_{n}^{k-m}\right)+V^{*} g\left(I^{*}\right) \ln \frac{V_{n}^{k+1-m} g\left(I_{n}^{k-m}\right)}{V_{n}^{k+1} g\left(I_{n}^{k}\right)} \\
& \left.+\left(S^{*} f\left(I^{*}\right)+V^{*} g\left(I^{*}\right)\right)\left(\frac{I_{n}^{k+1}}{I^{*}}-\frac{I_{n}^{k}}{I^{*}}+\ln \frac{I_{n}^{k}}{I^{*}}\right)\right] .
\end{aligned}
$$

When $n \in\{0,1, \ldots, M-1\}$, we have

$$
\frac{S_{n+1}^{k+1}}{S_{n}^{k+1}}-2+\frac{S_{n}^{k+1}}{S_{n+1}^{k+1}} \geq 0, \frac{V_{n+1}^{k+1}}{V_{n}^{k+1}}-2+\frac{V_{n}^{k+1}}{V_{n+1}^{k+1}} \geq 0 \text { and } \frac{I_{n+1}^{k+1}}{I_{n}^{k+1}}-2+\frac{I_{n}^{k+1}}{I_{n+1}^{k+1}} \geq 0 .
$$


Hence,

$$
\begin{aligned}
\Delta \bar{H}^{k} \leq & \frac{1}{(\Delta x)^{2}}\left[D_{1}\left(S_{M+1}^{k+1}-S_{M}^{k+1}+S_{-1}^{k+1}-S_{0}^{k+1}\right)-S^{*} D_{1}\left(\frac{S_{-1}^{k+1}}{S_{0}^{k+1}}-2+\frac{S_{M+1}^{k+1}}{S_{M}^{k+1}}\right)\right. \\
& +D_{2}\left(V_{M+1}^{k+1}-V_{M}^{k+1}+V_{-1}^{k+1}-V_{0}^{k+1}\right)-V^{*} D_{2}\left(\frac{V_{-1}^{k+1}}{V_{0}^{k+1}}-2+\frac{V_{M+1}^{k+1}}{V_{M}^{k+1}}\right) \\
& \left.+D_{4}\left(I_{M+1}^{k+1}-I_{M}^{k+1}+I_{-1}^{k+1}-I_{0}^{k+1}\right)-I^{*} D_{4}\left(\frac{I_{-1}^{k+1}}{I_{0}^{k+1}}-2+\frac{I_{M+1}^{k+1}}{I_{M}^{k+1}}\right)\right] \\
= & 0 .
\end{aligned}
$$

Thus,

$$
\begin{aligned}
& H^{k+1}-H^{k} \\
& \leq \sum_{n=0}^{M}\left[(\alpha+\mu) S^{*}\left(2-\frac{S^{*}}{S_{n}^{k+1}}-\frac{S_{n}^{k+1}}{S^{*}}\right)+(\mu+\beta) V^{*}\left(2-\frac{V^{*}}{V_{n}^{k+1}}-\frac{V_{n}^{k+1}}{V^{*}}\right)\right. \\
& +\alpha S^{*}\left(\frac{S_{n}^{k+1}}{S^{*}}+\frac{V^{*}}{V_{n}^{k+1}}-\frac{S_{n}^{k+1} V^{*}}{S^{*} V_{n}^{k+1}}-1\right) \\
& +\left(2-\frac{S^{*}}{S_{n}^{k+1}}+\frac{f\left(I_{n}^{k}\right)}{f\left(I^{*}\right)}-\frac{I^{*} S_{n}^{k+1-m} f\left(I_{n}^{k-m}\right)}{I_{n}^{k+1} S^{*} f\left(I^{*}\right)}-\frac{I_{n}^{k}}{I^{*}}+\ln \frac{I^{k} S_{n}^{k+1-m} f\left(I_{n}^{k-m}\right)}{I_{n}^{k+1} S_{n}^{k+1} f\left(I_{n}^{k}\right)}\right) S^{*} f\left(I^{*}\right) \\
& \left.+\left(2-\frac{V^{*}}{V_{n}^{k+1}}+\frac{g\left(I_{n}^{k}\right)}{g\left(I^{*}\right)}-\frac{I^{*} V_{n}^{k+1-m} g\left(I_{n}^{k-m}\right)}{I_{n}^{k+1} V^{*} g\left(I^{*}\right)}-\frac{I_{n}^{k}}{I^{*}}+\ln \frac{I^{k} V_{n}^{k+1-m} g\left(I_{n}^{k-m}\right)}{I_{n}^{k+1} V_{n}^{k+1} g\left(I_{n}^{k}\right)}\right) V^{*} g\left(I^{*}\right)\right] \\
& =-\sum_{n=0}^{M}\left[(\mu+\alpha) S^{*}\left(\Phi\left(\frac{S^{*}}{S_{n}^{k+1}}\right)+\Phi\left(\frac{S_{n}^{k+1}}{S^{*}}\right)\right)+(\mu+\beta) V^{*}\left(\Phi\left(\frac{V^{*}}{V_{n}^{k+1}}\right)+\Phi\left(\frac{V_{n}^{k+1}}{V^{*}}\right)\right)\right. \\
& -\alpha S^{*}\left(\Phi\left(\frac{S_{n}^{k+1}}{S^{*}}\right)+\Phi\left(\frac{V^{*}}{V_{n}^{k+1}}\right)-\Phi\left(\frac{S_{n}^{k+1} V^{*}}{S^{*} V_{n}^{k+1}}\right)\right) \\
& +S^{*} f\left(I^{*}\right)\left(\Phi\left(\frac{S^{*}}{S_{n}^{k+1}}\right)+\Phi\left(\frac{I^{*} S_{n}^{k+1-m} f\left(I_{n}^{k-m}\right)}{I_{n}^{k+1} S^{*} f\left(I^{*}\right)}\right)-\frac{f\left(I_{n}^{k}\right)}{f\left(I^{*}\right)}+\frac{I_{n}^{k}}{I^{*}}-\ln \frac{I_{n}^{k} f\left(I^{*}\right)}{I^{*} f\left(I_{n}^{k}\right)}\right) \\
& \left.+V^{*} g\left(I^{*}\right)\left(\Phi\left(\frac{V^{*}}{V_{n}^{k+1}}\right)+\Phi\left(\frac{I^{*} V_{n}^{k+1-m} g\left(I_{n}^{k-m}\right)}{I_{n}^{k+1} V^{*} g\left(I^{*}\right)}\right)-\frac{g\left(I_{n}^{k}\right)}{g\left(I^{*}\right)}+\frac{I_{n}^{k}}{I^{*}}-\ln \frac{I_{n}^{k} g\left(I^{*}\right)}{I^{*} g\left(I_{n}^{k}\right)}\right)\right] \\
& =-\sum_{n=0}^{M}\left[\mu S^{*}\left(\Phi\left(\frac{S^{*}}{S_{n}^{k+1}}\right)+\Phi\left(\frac{S_{n}^{k+1}}{S^{*}}\right)\right)+\alpha S^{*}\left(\Phi\left(\frac{S^{*}}{S_{n}^{k+1}}\right)+\Phi\left(\frac{S_{n}^{k+1} V^{*}}{S^{*} V_{n}^{k+1}}\right)\right)\right. \\
& +\mu q A \Phi\left(\frac{V^{*}}{V_{n}^{k+1}}\right)+(\mu+\beta) V^{*} \Phi\left(\frac{V_{n}^{k+1}}{V^{*}}\right) \\
& +S^{*} f\left(I^{*}\right)\left(\Phi\left(\frac{S^{*}}{S_{n}^{k+1}}\right)+\Phi\left(\frac{I^{*} S_{n}^{k+1-m} f\left(I_{n}^{k-m}\right)}{I_{n}^{k+1} S^{*} f\left(I^{*}\right)}\right)-\frac{f\left(I_{n}^{k}\right)}{f\left(I^{*}\right)}+\frac{I_{n}^{k}}{I^{*}}-\ln \frac{I_{n}^{k} f\left(I^{*}\right)}{I^{*} f\left(I_{n}^{k}\right)}\right) \\
& \left.+V^{*} g\left(I^{*}\right)\left(\Phi\left(\frac{I^{*} V_{n}^{k+1-m} g\left(I_{n}^{k-m}\right)}{I_{n}^{k+1} V^{*} g\left(I^{*}\right)}\right)-\frac{g\left(I_{n}^{k}\right)}{g\left(I^{*}\right)}+\frac{I_{n}^{k}}{I^{*}}-\ln \frac{I_{n}^{k} g\left(I^{*}\right)}{I^{*} g\left(I_{n}^{k}\right)}\right)\right] .
\end{aligned}
$$

Applying Assumption (H2) and that $\ln x \leq x-1$, we can get

$$
\frac{\mathcal{G}\left(I_{n}^{k}\right)}{\mathcal{G}\left(I^{*}\right)}-\frac{I_{n}^{k}}{I^{*}}+\ln \left(\frac{I_{n}^{k} \mathcal{G}\left(I^{*}\right)}{I^{*} \mathcal{G}\left(I_{n}^{k}\right)}\right) \leq \frac{\mathcal{G}\left(I_{n}^{k}\right)}{\mathcal{G}\left(I^{*}\right)}-\frac{I_{n}^{k}}{I^{*}}+\frac{I_{n}^{k} \mathcal{G}\left(I^{*}\right)}{I^{*} \mathcal{G}\left(I_{n}^{k}\right)}-1 \leq 0,
$$


where $\mathcal{G}=\{f, g\}$. Therefore,

$$
\begin{aligned}
H^{k+1}-H^{k} \leq & -\sum_{n=0}^{M}\left[\mu S^{*}\left(\Phi\left(\frac{S^{*}}{S_{n}^{k+1}}\right)+\Phi\left(\frac{S_{n}^{k+1}}{S^{*}}\right)\right)+\alpha S^{*}\left(\Phi\left(\frac{S^{*}}{S_{n}^{k+1}}\right)+\Phi\left(\frac{S_{n}^{k+1} V^{*}}{S^{*} V_{n}^{k+1}}\right)\right)\right. \\
& +\mu q A \Phi\left(\frac{V^{*}}{V_{n}^{k+1}}\right)+(\mu+\beta) V^{*} \Phi\left(\frac{V_{n}^{k+1}}{V^{*}}\right) \\
& +S^{*} f\left(I^{*}\right)\left(\Phi\left(\frac{S^{*}}{S_{n}^{k+1}}\right)+\Phi\left(\frac{I^{*} S_{n}^{k+1-m} f\left(I_{n}^{k-m}\right)}{I_{n}^{k+1} S^{*} f\left(I^{*}\right)}\right)\right) \\
& \left.+V^{*} g\left(I^{*}\right) \Phi\left(\frac{I^{*} V_{n}^{k+1-m} g\left(I_{n}^{k-m}\right)}{I_{n}^{k+1} V^{*} g\left(I^{*}\right)}\right)\right] \leq 0 .
\end{aligned}
$$

Clearly, $H^{k}$ is a non-increasing sequence. There exists $\mathcal{H}>0$ such that $\lim _{k \rightarrow \infty} H^{k}=\mathcal{H}$, yielding that $\lim _{k \rightarrow \infty}\left(H^{k+1}-H^{k}\right)=0$. This means that $\lim _{k \rightarrow \infty} S_{n}^{k}=S^{*}, \lim _{k \rightarrow \infty} V_{n}^{k}=V^{*}, \lim _{k \rightarrow \infty} I_{n}^{k}=I^{*}$.

\section{$5 \quad$ Numerical Simulations}

From (3.1), set $f(I)=\frac{\beta_{1} I}{1+I}, g(I)=\frac{\beta_{2} I}{1+I}, \Delta x=0.2$ and $\Delta t=0.1$. Referring to [13, 26], we take $\delta=0, p=1, q=0, D_{1}=D_{2}=D_{4}=D=1$ and the other parameters as follows:

$$
\beta_{1}=0.0004, \beta_{2}=0.000012, A=175000, \mu=0.04, \beta=0.05, \gamma=0.02 \text {. }
$$

Case 1 Choose $\alpha=9, \tau=20$ and initial condition

$$
\begin{aligned}
& S(n, k)=100 \sin n+100, V(n, k)=7000(1+\cos n), \\
& I(n, k)=\sin (0.5 n)+1, n \in\{0,1, \ldots, 200\}, k \in\{-m,-m+1, \ldots, 0\} .
\end{aligned}
$$

By $(2.3)$ and simple calculations, we have that $\mathcal{R}_{0}=0.9278<1$ and that $E_{0}=(77.4336$, $7743.3628,0)$. Using Theorem 4.1, $E_{0}$ is globally asymptotically stable. One gets that the disease is extinct (see Figure 1).
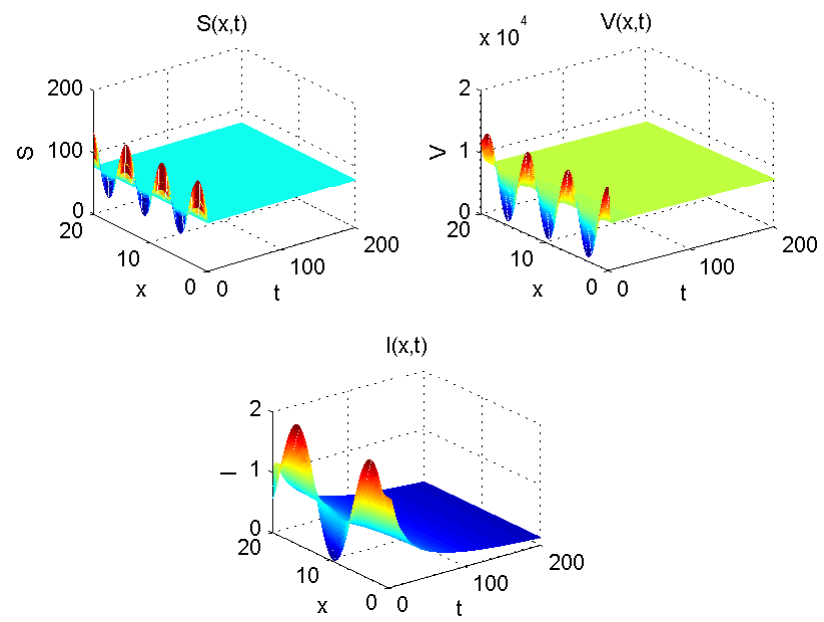

Figure 1 The disease-free equilibrium $E_{0}=(77.4336,7743.3628,0)$ of system (3.1) is globally asymptotically stable when $\mathcal{R}_{0}=0.9278<1$ 
Case 2 Choose $\alpha=0.9, \tau=20$ and initial condition

$$
\begin{gathered}
S(n, k)=700 \sin n+700, V(n, k)=8000(1+\cos n), \\
I(n, k)=\sin (0.5 n)+2, n \in\{0,1, \ldots, 200\}, k \in\{-m,-m+1, \ldots, 0\} .
\end{gathered}
$$

We obtain that $\mathcal{R}_{0}=2.8999>1$ and that $E^{*}=(744.4733,7444.0831,1.8991)$, respectively. Thus, $E^{*}$ is globally asymptotically stable, by Theorem 4.2 . Hence, the disease will eventually become endemic (see Figure 2).
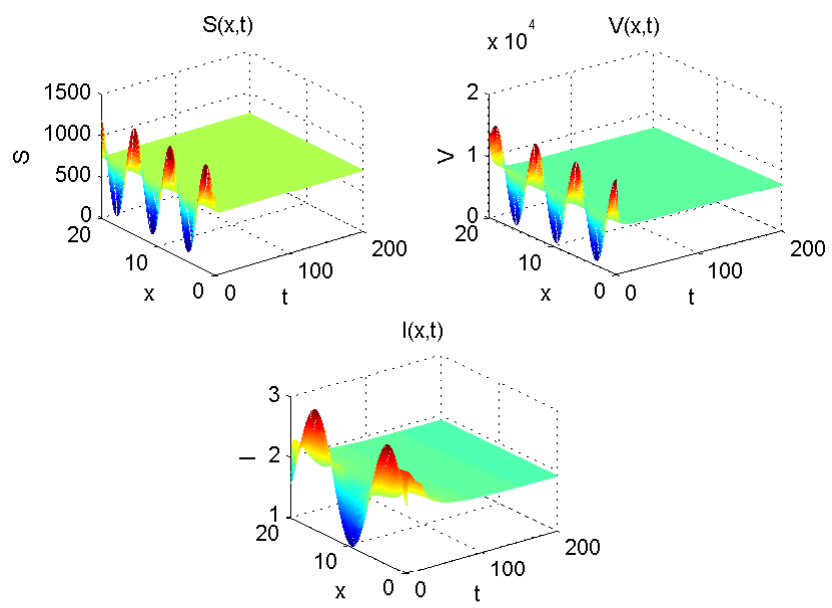

Figure 2 The disease-free equilibrium $E^{*}=(744.4733,7444.0831,1.8991)$ of system (3.1) is globally asymptotically stable when $\mathcal{R}_{0}=2.8999>1$

Case 3 Effect of time delay.

Choose $\tau=5,10,15,20$ with $\alpha=0.9$ and an initial condition as in Case (2). We obtain that $\mathcal{R}_{0}=5.2840,4.3262,3.5420,2.8999$ and that $I^{*}=4.2821,3.3247,2.5408,1.8991$, respectively. Here, we give the simulations of solutions of the infectious $I$ at $x=10$ with different values of $\tau$. We observe that the number of those who are infectious decreases with an increase of $\tau$ (see Figure 3). Biologically, this delay can play an important role in eliminating the number of people who are infectious. By increasing the delay, we can decrease the number of people who are infectious.

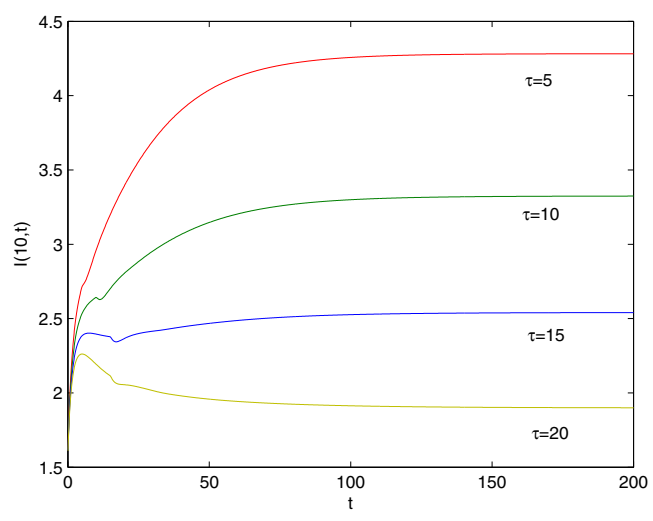

Figure 3 The solutions of the infectious $I$ at $x=10$ with different $\tau$ in Case (3) 


\section{Conclusions}

In this paper, we proposed a diffusive SVEIR epidemic model with time delay and general incidence. For this model, we first considered the global dynamics of the continuous case. Then, by using the NSFD scheme, we derived the discretization of the model. It has been shown that the global stability of the equilibria is completely determined by the basic reproduction number $\mathcal{R}_{0}$ : if $\mathcal{R}_{0} \leq 1$, then the disease-free equilibrium $E_{0}$ is globally asymptotically stable; if $\mathcal{R}_{0}>1$, then the endemic equilibrium $E^{*}$ is globally asymptotically stable. One sees that the NSFD scheme can preserve the global properties of solutions for an original continuous model, such as the positivity and ultimate boundedness of solutions, and global stability of the equilibria. It is our intention to use this method to study other delayed diffusive epidemic models.

\section{References}

[1] Kribs-Zaleta C M, Velasco-Hernández J X. A simple vaccination model with multiple endemic states. Mathematical Biosciences, 2000, 164(2): 183-201

[2] Arino J, McCluskey C C, van den Driess P. Global results for an epidemic model with vaccination that exhibits backward bifurcation. SIAM Journal on Applied Mathematics, 2003, 64(1): 260-276

[3] Li J, Ma Z, Zhou Y. Global analysis of SIS epidemic model with a simple vaccination and multiple endemic equilibria. Acta Mathematica Scientia, 2006, 26B(1): 83-93

[4] Liu X, Takeuchi Y, Iwami S. SVIR epidemic models with vaccination strategies. Journal of Theoretical Biology, 2008, 253(1): 1-11

[5] Li J, Yang Y. SIR-SVS epidemic models with continuous and impulsive vaccination strategies. Journal of Theoretical Biology, 2011, 280(1): 108-116

[6] Zhu Q, Hao Y, Ma J, Yu S, Wang Y. Surveillance of hand, foot, and mouth disease in mainland china (2008-2009). Biomedical and Environmental Sciences, 2011, 24(4): 349-356

[7] Martcheva M. Avian flu: modeling and implications for control. Journal of Biological Systems, 2014, 22(1): $151-175$

[8] Wang W, Ruan S. Simulating the SARS outbreak in beijing with limited data. Journal of Theoretical Biology, 2004, 227(3): 369-379

[9] Liljeros F, Edling C R, Amaral L A N. Sexual networks: implications for the transmission of sexually transmitted infections. Microbes and Infection, 2003, 5(2): 189-196

[10] Liu W M, Levin S A, Iwasa Y. Influence of nonlinear incidence rates upon the behavior of SIRS epidemiological model. Journal of Mathematical Biology, 1986, 23(2): 187-204. 18

[11] Capasso V, Serio G. A generalization of the Kermack-McKendrick deterministic epidemic model. Mathematical Biosciences, 1978, 42(1/2): 43-61

[12] Hattaf K, Lashari A A, Louartassi Y, Yousfi N. A delayed SIR epidemic model with general incidence rate. Electronic Journal of Qualitative Theory of Differential Equations, 2013(3): 1-9

[13] Wang L, Liu Z, Zhang X. Global dynamics of an SVEIR epidemic model with distributed delay and nonlinear incidence. Applied Mathematics and Computation, 2016, 284: 47-65

[14] Hattaf K. Global stability and Hopf bifurcation of a generalized viral infection model with multi-delays and humoral immunity. Physica A: Statal Mechanics and its Applications, 2020, 545: 123689

[15] Wang J, Huang G, Takeuchi Y, Liu S. SVEIR epidemiological model with varying infectivity and distributed delays. Mathematical Biosciences and Engineering, 2011, 8(3): 875-888

[16] Xu R. Global stability of a delayed epidemic model with latent period and vaccination strategy. Applied Mathematical Modelling, 2012, 36(11): 5293-5300

[17] Duan X, Yuan S, Li X. Global stability of an SVIR model with age of vaccination. Applied Mathematics and Computation, 2014, 226: 528-540

[18] Meng X, Chen L, Wu B. A delay SIR epidemic model with pulse vaccination and incubation times. Nonlinear Analysis: Real World Applications, 2010, 11(1): 88-98

[19] Gao S, Chen L, Nieto J J, Torres A. Analysis of a delayed epidemic model with pulse vaccination and saturation incidence. Vaccine, 2006, 24(35/36): 6037-6045 
[20] Zhang T, Zhang T Q, Meng X. Stability analysis of a chemostat model with maintenance energy. Applied Mathematics Letters, 2017, 68: 1-7

[21] Zhang T, Liu X, Meng X, Zhang T Q. Spatio-temporal dynamics near the steady state of a planktonic system. Computers and Mathematics with Applications, 2018, 75(12): 4490-4504

[22] Hattaf K, Yousfi N. Global stability for reaction-diffusion equations in biology. Computers Mathematics with Applications, 2013, 66(8): 1488-1497

[23] Webby R J, Webster R G. Are we ready for pandemic influenza? Science, 2003, 302: 1519-1522

[24] Xu Z, Ai C. Traveling waves in a diffusive influenza epidemic model with vaccination. Applied Mathematical Modelling, 2016, 40(15/16): 7265-7280

[25] Abdelmalek S, Bendoukha S. Global asymptotic stability of a diffusive SVIR epidemic model with immigration of individuals. Electronic Journal of Differential Equations, 2016, 2016(129/324): 1-14

[26] Xu Z, Xu Y, Huang Y. Stability and traveling waves of a vaccination model with nonlinear incidence. Computers and Mathematics with Applications, 2018, 75(2): 561-581

[27] Mickens R E. Nonstandard Finite Difference Models of Differential Equations. World Scientific, December 1993

[28] Arenas A J, Morano J A, Cortés J C. Non-standard numerical method for a mathematical model of RSV epidemiological transmission. Computers and Mathematics with Applications, 2008, 56(3): 670-678

[29] Hattaf K, Yousfi N. Global properties of a discrete viral infection model with general incidence rate. Mathematical Methods in the Applied Sciences, 2016, 39(5): 998-1004

[30] Hattaf K, Yousfi N. A numerical method for delayed partial differential equations describing infectious diseases. Computers and Mathematics with Applications, 2016, 72(11): 2741-2750

[31] Liu J, Peng B, Zhang T. Effect of discretization on dynamical behavior of SEIR and SIR models with nonlinear incidence. Applied Mathematics Letters, 2015, 39: 60-66

[32] Muroya Y, Bellen A, Enatsu Y, Nakata Y. Global stability for a discrete epidemic model for disease with immunity and latency spreading in a heterogeneous host population. Nonlinear Analysis: Real World Applications, 2013, 13(1): 258-274

[33] Qin W, Wang L, Ding X. A non-standard finite difference method for a hepatitis B virus infection model with spatial diffusion. Journal of Difference Equations and Applications, 2014, 20(12): 1641-1651

[34] Geng Y, Xu J. Stability preserving NSFD scheme for a multi-group SVIR epidemic model. Mathematical Methods in the Applied Sciences, 2017, 40(13): 4917-4927

[35] Ding D, Ma Q, Ding X. A non-standard finite difference scheme for an epidemic model with vaccination. Journal of Difference Equations and Applications, 2013, 19(2): 179-190

[36] Yang Y, Zhou J, Ma X, Zhang T. Nonstandard finite difference scheme for a diffusive within-host virus dynamics model with both virus-to-cell and cell-to-cell transmissions. Computers and Mathematics with Applications, 2016, 72(4): 1013-1020

[37] Zhou J, Yang Y, Zhang T. Global dynamics of a reaction-diffusion waterborne pathogen model with general incidence rate. Journal of Mathematical Analysis and Applications, 2018, 466(1): 835-859

[38] Martin R H, Smith H L. Abstract functional differential equations and reaction-diffusion systems. Transactions of the American Mathematical Society, 1990, 321(1): 1-44

[39] Fujimoto T, Ranade R R. Two characterizations of inverse-positive matrices: the Hawkins-Simon condition and the Le Chatelier-Braun principle. The Electronic Journal of Linear Algebra, 2004, 11: 59-65 